\title{
A Comprehensive Strategy to Quantify the Complex System by Ultraviolet and Infrared Spectra Analyses Coupled with Combustion Heat for Recognizing the Quality Consistency of San-Huang Tablets
}

\author{
Yan Wang1,2, Guoxiang Sun 1*, Yang Jin³ , Jing Zhang', Jiayue Gao1, \\ Eboka Majolene Benedicta Sabi-Mouka ${ }^{4}$ \\ ${ }^{1}$ College of Pharmacy, Shenyang Pharmaceutical University, Shenyang, China \\ ${ }^{2}$ College of Pharmacy and Chemistry, Dali University, Dali, China \\ ${ }^{3}$ Department of Pharmacy, Traditional Chinese Medicine Hospital of Dali, Dali, China \\ ${ }^{4}$ Department of Pharmaceutical Analysis, China Pharmaceutical University, Nanjing, China \\ Email: ${ }^{\star}$ gxswmwys@163.com
}

How to cite this paper: Wang, Y., Sun, G.X., Jin, Y., Zhang, J., Gao, J.Y. and Sabi-Mouka, E.M.B. (2017) A Comprehensive Strategy to Quantify the Complex System by Ultraviolet and Infrared Spectra Analyses Coupled with Combustion Heat for Recognizing the Quality Consistency of San-Huang Tablets. American Journal of Analytical Chemistry, 8, 668-680. https://doi.org/10.4236/ajac.2017.810049

Received: August 31, 2017

Accepted: October 27, 2017

Published: October 30, 2017

Copyright (c) 2017 by authors and Scientific Research Publishing Inc. This work is licensed under the Creative Commons Attribution International License (CC BY 4.0).

http://creativecommons.org/licenses/by/4.0/

\begin{abstract}
Spectral quantitative fingerprinting including ultraviolet (UV) and Fourier transform infrared (FT-IR) coupled with combustion heat $(\mathrm{CH})$ analytical techniques was employed and compared for rapid screening quality grade and discriminating San-Huang Tablets (SHT) of different commercial brands. The systematic quantified fingerprint method (SQFM) was applied to evaluate, qualitatively and quantitatively, the quality consistency of the herbal preparation. It was possible to deduce that the quantitative similarity analysis by SQFM was enabled to make a good discrimination of the tested samples. It was a particularly useful method for the overall quality evaluation of herbal medicine and their preparations.
\end{abstract}

\section{Keywords}

Combustion Heat, San-Huang Tablets, Spectral Fingerprint, Systematic Quantified Fingerprint Method

\section{Introduction}

Traditional Chinese medicine (TCM) and herbal drugs (HD) are very popular in different systems of medicines such as Chinese medicine, naturopathy and homeopathy. They play increasingly important roles in healthcare of the majority 
of the population worldwide [1]. Traditionally, one or two markers' determination is performed on TCM identification [2], and such approach might be unreliable and far from satisfactory. As the therapeutic effects of TCM and HD are based on the complex interaction of numerous ingredients, they are different from those of synthetic drugs [3].

Up to now, chemical fingerprint techniques have already been internationally acknowledged and employed by existing regulations and guidelines such as the World Health Organization, the American Food and Drug Administration and The Chinese State Food and Drug Administration. Meanwhile, multiple kinds of techniques such as high performance liquid chromatography in combination with different detectors, gas chromatography, nuclear magnetic resonance [4] [5] [6] have been successfully applied to establish fingerprints for quality control of TCM and HD. Though the reported results evidenced the feasibility of these methodologies, it should be pointed out that there are also some disadvantages with respect to them. For instance, source identification requires complicated peak matching techniques, which lead to more challenges in data processing and fingerprint comparison. Meanwhile, other rests of the proposed strategies need expensive equipment and laborious sample preparation prior to analysis. Therefore, spectroscopy techniques such as ultraviolet (UV), infrared (IR), near infrared (NIR) [7] [8] [9] [10] with the characteristics of fast analysis, non-destructiveness, low equipment cost have become powerful analytical tools.

In addition, most published reports evaluated the similarity between sample fingerprint (SFP) and reference fingerprint (RFP) only qualitatively [11] [12], but quantitative fingerprint evaluation can provide a more comprehensive view of the fingerprints not only based on the similarity of peak distribution, but also the quantitative content of the peaks.

The Chinese Pharmacopoeia lists the monograph of San-Huang Tablets (SHT), and there are also some literature reports regarding the qualitative and quantitative study of SHT [13] [14], but studies with respect to the fast quality analysis can rarely be found. Therefore, in this experiment, SHT, the most popular herbal formulation for the treatment of gingival bleeding, intense heat in the body, reddish urine and constipation in clinical practice [15], was taken as an example to propose a comprehensive strategy for its quality consistency monitoring. The aim of the study was to establish visible spectral quantitative fingerprints combined UV with IR. Moreover, the combustion heat $(\mathrm{CH})$ frequently used in physical chemistry was employed for the first time to reflect the whole chemical information of SHT. Finally, the integrated assessment method involving UV and IR quantitative fingerprints coupled with $\mathrm{CH}$ based on systematic quantified fingerprint method (SQFM) was applied and analyzed the quality consistency of samples from batch to batch and manufactory to manufactory.

\section{Materials and Methods}

\subsection{Chemicals and Materials}

A total of thirty batches of SHT samples (labeled S1-S30) from nineteen manu- 
facturers were listed in Table 1. Methanol (purchased from Yuwang Industry Limited Company, Shandong, China) was HPLC grade. The other reagents were all analytical grade.

\subsection{Sample Preparation}

Ten tablets of SHT were accurately weighed to get the average weight for each one. A quantity equivalent to two tablets in powdered states was weighed and extracted with $20 \mathrm{~mL}$ methanol in an ultrasonic water bath for $20 \mathrm{~min}$. The

Table 1. The source of 30 San-Huang Tablets samples in this study.

\begin{tabular}{|c|c|c|}
\hline & & Source \\
\hline Sample no. & Batch no. & Manufacturer \\
\hline S1 & D95015 & Baishang Tangwei Pharmaceutical Co., Ltd. \\
\hline $\mathrm{S} 2$ & D95019 & Baishang Tangwei Pharmaceutical Co., Ltd. \\
\hline S3 & 110301 & Anhui Renhe Pharmaceutical Co., Ltd. \\
\hline $\mathrm{S} 4$ & 120506 & Guangxi Banyutianlong Pharmaceutical Co., Ltd. \\
\hline S5 & 111195 & Handan Moluodan Pharmaceutical Co., Ltd. \\
\hline S6 & D15045 & Hebei Shijitangwei Pharmaceutical Co., Ltd. \\
\hline S7 & 11104 & Henan Fusen Pharmaceutical Co., Ltd. \\
\hline S8 & 120401 & Henan Huaiqing Pharmaceutical Co., Ltd. \\
\hline S9 & 20120401 & Henan Kangqi Pharmaceutical Co., Ltd. \\
\hline S10 & 20120301 & Henan Jishi Pharmaceutical Co., Ltd. \\
\hline S11 & KL100939 & Jindu Nianantang Pharmaceutical factory \\
\hline S12 & 110303 & Luoyan Junsan Pharmaceutical Co., Ltd. \\
\hline S13 & 120101 & Luoyan Junsan Pharmaceutical Co., Ltd \\
\hline S14 & 120501 & Luoyan Junsan Pharmaceutical Co., Ltd. \\
\hline S15 & 120401 & Luoyan Junsan Pharmaceutical Co., Ltd. \\
\hline S16 & 110601 & Guangxi Banyutianlong Pharmaceutical Co., Ltd. \\
\hline S17 & 110901 & Shandong Jianming Pharmaceutical Co., Ltd. \\
\hline $\mathrm{S} 18$ & 20111105 & Sanxi Hetai Pharmaceutical Co., Ltd. \\
\hline S19 & 110304 & Sanxi Hetai Pharmaceutical Co., Ltd. \\
\hline S20 & 20120404 & Sanxi Hetai Pharmaceutical Co., Ltd. \\
\hline $\mathrm{S} 21$ & 120502 & Shanxi Lijun Pharmaceutical Co., Ltd. \\
\hline $\mathrm{S} 22$ & 100805 & Xiangfan Longzhong Pharmaceutical Co., Ltd. \\
\hline S23 & 131201 & Guangxi Banyutianlong Pharmaceutical Co., Ltd. \\
\hline $\mathrm{S} 24$ & 20120401 & Xinxiang Zuojinming Pharmaceutical Co., Ltd. \\
\hline S25 & 110321 & Yabao Pharmaceutical Co., Ltd. \\
\hline S26 & 111122 & Yabao Pharmaceutical Co., Ltd. \\
\hline S27 & 120212 & Yabao Pharmaceutical Co., Ltd. \\
\hline S28 & 20100902 & Zhengzhou Yumi Pharmaceutical Co., Ltd. \\
\hline S29 & 20120502 & Xinxiang Zuojinming Pharmaceutical Co., Ltd. \\
\hline $\mathrm{S} 30$ & 110801 & Hubei Wudang Pharmaceutical Co., Ltd. \\
\hline
\end{tabular}


extracted solution was filtered and then diluted to $25 \mathrm{~mL}$ in a volumetric flask with methanol. The solution was centrifuged at $3500 \mathrm{rpm}$ for $10 \mathrm{~min}$, and the supernatant obtained was filtered through a $0.45 \mu \mathrm{m}$ Millipore filter for UV spectroscopic analysis.

$1 \mathrm{mg}$ of the powdered and dried SHT was weighed, then $100 \mathrm{mg}$ of $\mathrm{KBr}$ powder was added. After thorough mixing with an agate mortar, they were pressed into a $\mathrm{KBr}$ crystal tablet for IR spectroscopic analysis.

\subsection{Analysis Conditions}

Flow injection analysis (FIA) was employed, and the unseparated chromatograms of UV spectra were recorded on an Agilent 1100 HPLC series (Hewlett Packard, CA), coupled with an Agilent polytetrafluoroethylene tube $(6500 \mathrm{~mm} \times$ $0.12 \mathrm{~mm}$ ) and a DAD detector (190 - $400 \mathrm{~nm}$, acquired at $1 \mathrm{~nm}$ intervals). The column temperature was set at $30.00( \pm 0.15)^{\circ} \mathrm{C}$. The mobile phase was $100 \%$ $(\mathrm{v} / \mathrm{v})$ methanol at a flow rate of $0.8 \mathrm{~mL} / \mathrm{min}$ with an injection volume of $0.2 \mu \mathrm{L}$. FIA method (specified in Figure 1), which used a packless column instead of the reversed phase column and detected the UV signal by DAD.

The Fourier transformed infrared (FT-IR) scans were performed on a Bruker IFS-55 type Fourier infrared spectral instrument equipped with a deuterated triglycine sulfate (DTGS) detector (BRUGG GROUP). Spectral scans were recorded between 4000 and $400 \mathrm{~cm}^{-1}$ at $8 \mathrm{~cm}^{-1}$ resolution. The spectra were converted to ASCII format and transferred to an Excel file for statistical analysis.

$1 \mathrm{~g}$ of the dried SHT was weighed and performed on a ZDHW6000 computer automatic calorimeter (Hebi ying hua instruments limited company, China) for $\mathrm{CH}$ analysis.

\subsection{Data Analysis}

All original data acquired were processed by a ChemStation workstation (Agilent technology Inc.). Similarity analysis of UV and IR fingerprints based on

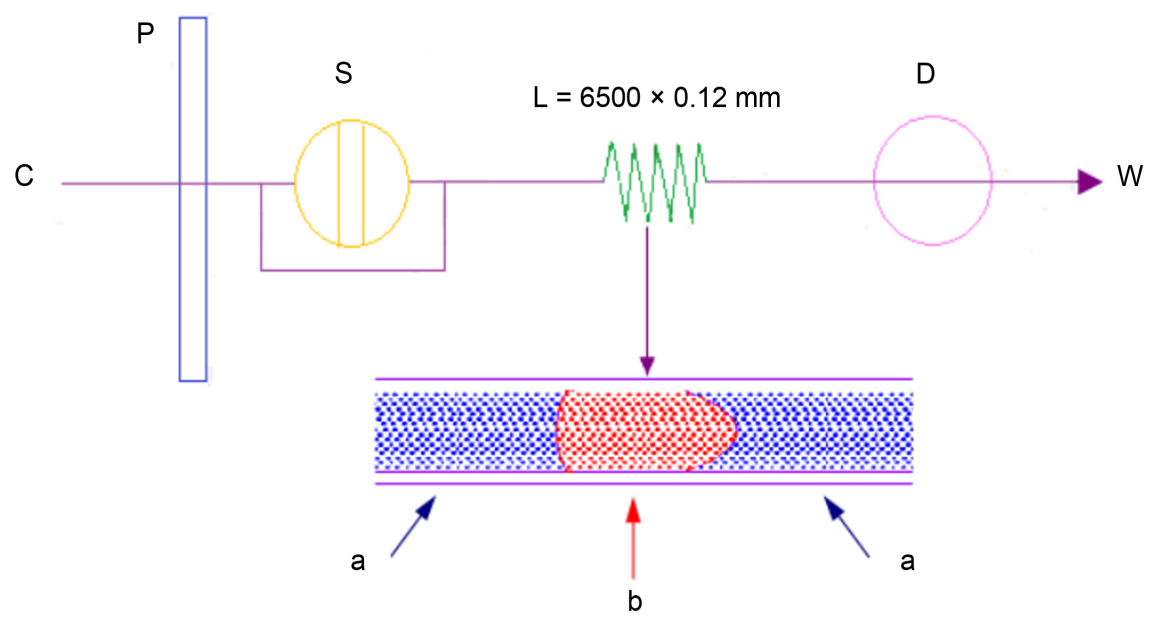

Figure 1. Diagram of FIA method. C: carrier; P: pump; S: sample injector; L: hollow pipe; D: diode array detector; W: disposal bottle; a: carrier solution; b: sample solution. 
SQFM was performed on software 3.0 of Digitized Evaluation System for Super-Information Characteristics of UV Fingerprints (software Certificate No: 0462756, China) and software 4.0 of Digitized Evaluation System for Super-Information Characteristics of IR Fingerprints (software Certificate No: 0474079, China), respectively.

\subsection{Theory}

The qualitative similarity $S$, describes in Equation (1) can monitor the number of chemical fingerprint and the distribution proportion between SFP and RFP. The $S$ ranges between 0 and 1 , with 0 meaning no similarity between the fingerprints and 1 identical fingerprints. The quantitative similarity $P$ describes in Equation (2) can monitor the overall content of chemical fingerprints of the system. Relative deviation $\alpha$ (in Equation (3) defines as the relative deviation of leveling coefficient is proposed to discover the similarity between SFP and RFP. From Equation (1) to Equation (3), $n$ represents the fingerprint peak number, $x_{\mathrm{i}}$ and $y_{\mathrm{i}}$ is the peak area of the $i$ th common constituent existing in sample fingerprint vector $\boldsymbol{x}=\left(x_{1}, x_{2}, \cdots, x_{n}\right)$ and reference fingerprint vector $\boldsymbol{y}=\left(y_{1}, y_{2}, \cdots, y_{n}\right)$, respectively. SQFM, is a method combining $S$ with $P$ and $\alpha$ to simultaneously determine or identify the quality level of TCM, and the quality is divided into 8 grades in terms of SQFM criterion (summarized in Table 2).

$$
\begin{gathered}
S=\frac{1}{2}\left(S_{\mathrm{F}}+S_{\mathrm{F}}^{\prime}\right)=\frac{1}{2}\left(\frac{\sum_{i=1}^{n} x_{i} y_{i}}{\sqrt{\sum_{i=1}^{n} x_{i}^{2}} \sqrt{\sum_{i=1}^{n} y_{i}^{2}}}+\frac{\sum_{i=1}^{n} \frac{x_{i}}{y_{i}}}{\sqrt{n \sum_{i=1}^{n}\left(\frac{x_{i}}{y_{i}}\right)^{2}}}\right) \\
P=\frac{1}{2}(C+P) \\
=\frac{1}{2}\left(\frac{\sum_{i=1}^{n} x_{i} y_{i}}{\sum_{i=1}^{n} y_{i}^{2}}+\frac{\sum_{i=1}^{n} x_{i}}{\sum_{i=1}^{n} y_{i}} S_{\mathrm{F}}\right) \times 100 \% \\
\alpha=\left|1-\frac{\gamma_{x}}{\gamma_{y}}\right|=\left|1-\frac{P}{C}\right|
\end{gathered}
$$

Table 2. The quality grade identified by SQFM.

\begin{tabular}{ccccccccc}
\hline Para. & I & II & III & IV & V & VI & VII & VIII \\
\hline $\boldsymbol{S} \geq$ & $0.95-1.00$ & $0.90-0.95$ & $0.85-0.90$ & $0.80-0.85$ & $0.70-0.80$ & $0.60-0.70$ & $0.50-0.60$ & $<0.5$ \\
$P / \%$ & $95-105$ & $90-110$ & $80-120$ & $75-125$ & $70-130$ & $60-140$ & $50-150$ & $0-\infty$ \\
$\alpha<$ & 0.05 & 0.10 & 0.15 & 0.20 & 0.30 & 0.40 & 0.50 & $>0.50$ \\
Quality & best & better & good & fine & moderate & common & defective inferior \\
\hline
\end{tabular}




\section{Results and Discussions}

\subsection{UV Fingerprint}

\subsubsection{Methodology Validation}

Unseparated chromatograms at $254 \mathrm{~nm}$ and UV spectra in the region of 190 $400 \mathrm{~nm}$ were recorded, the retention time (RT) and peak area (PA) of unseparated chromatograms of samples were used to estimate the repeatability, precision and stability. The repeatability test was determined by analyzing six replicates of the same batch of sample, and the relative standard deviation (RSD) of $\mathrm{RT}$ and PA were less than $1.0 \%$ and $5.0 \%$, respectively. Instrument precision was determined by injecting the same sample solution six times consecutively, and the RSD of RT and PA were found to be $0.4 \%$ and $3.3 \%$, respectively. The solution stability was measured by testing a single sample solution stored at room temperature for $0,0.25,0.5,1,2$ and 4 hours, and the RSD of RT and PA did not exceed $1.0 \%$ and $3.0 \%$, respectively.

\subsubsection{Spectrum Analysis}

All the thirty samples were extracted under the optimized conditions and analyzed using the above-established method. The UV absorption spectra of the tested samples from 190 to $400 \mathrm{~nm}$ were revealed in Figure 2(a). The variation of the thirty samples was significant especially from 210 to $280 \mathrm{~nm}$, and the majority of components with chemical bonds $\pi \rightarrow \pi^{*}, n \rightarrow \pi^{*}$ in structure may contribute to the strong absorption at around $215 \mathrm{~nm}, 275 \mathrm{~nm}$ and $355 \mathrm{~nm}$ (Figure 2(c)), such as flavonoids and anthraquinones components in SHT.

\subsubsection{Fingerprint Analysis in Terms of SQFM}

The UV spectra of the collected samples and the RFP obtained by average method were converted into the file layout of CSV, and then imported into the in-house software. Similarity parameters $\left(S_{\mathrm{UV}}, P_{\mathrm{UV}}, \alpha_{\mathrm{UV}}\right)$ and final quality grades were calculated in Table 3 , and it was indicated that all samples were almost similar to the RFP with qualitative similarity $S_{\mathrm{UV}} \geq 0.93$. However, a great difference was observed in quantitative similarity $P_{\mathrm{UV}}$, which made an obvious distinction of all samples. The results in Table 3 shown that most of the samples were qualified (grade $\leq 5$ ) [13] except S8, S12, S18, S28, S30 for their higher contents in the range of $145.5 \%-253.8 \%$, and S17, S21, S22, S25, S27 for their lower contents in the range of $46.3 \%-67.5 \%$. Moreover, S1 and S2 from the same manufactory were proved to be accordant in quality of grade 2 and 3, respectively. However, S23 and S24 from the same vendors, got the inconsistent results of grade 1 and 5 , respectively.

\subsection{IR Fingerprint}

\subsubsection{Methodology Validation}

The repeatability test was determined by analyzing six replicates of the same batch of sample. The qualitative similarity $S_{\mathrm{IR}}$ and RSD were calculated, and the results showed that $S_{\mathrm{IR}} \geq 0.99$ with $\mathrm{RSD} \leq 3.0 \%$. Instrument precision was 


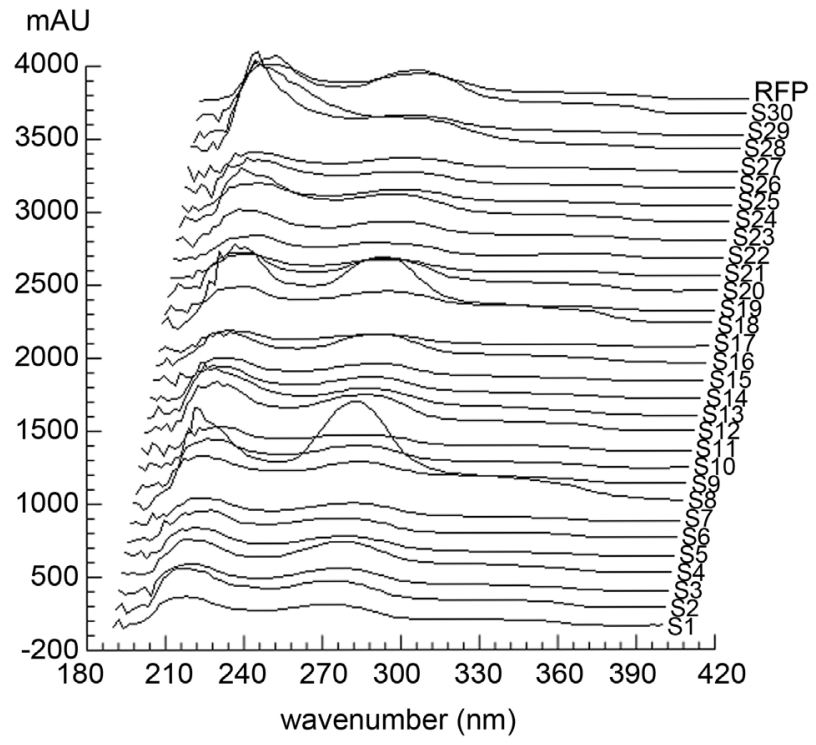

(a)

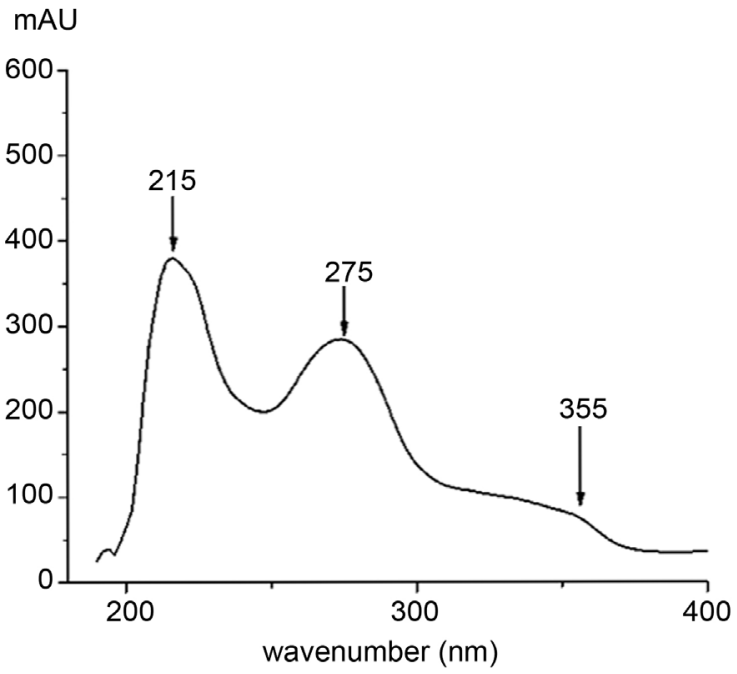

(c)

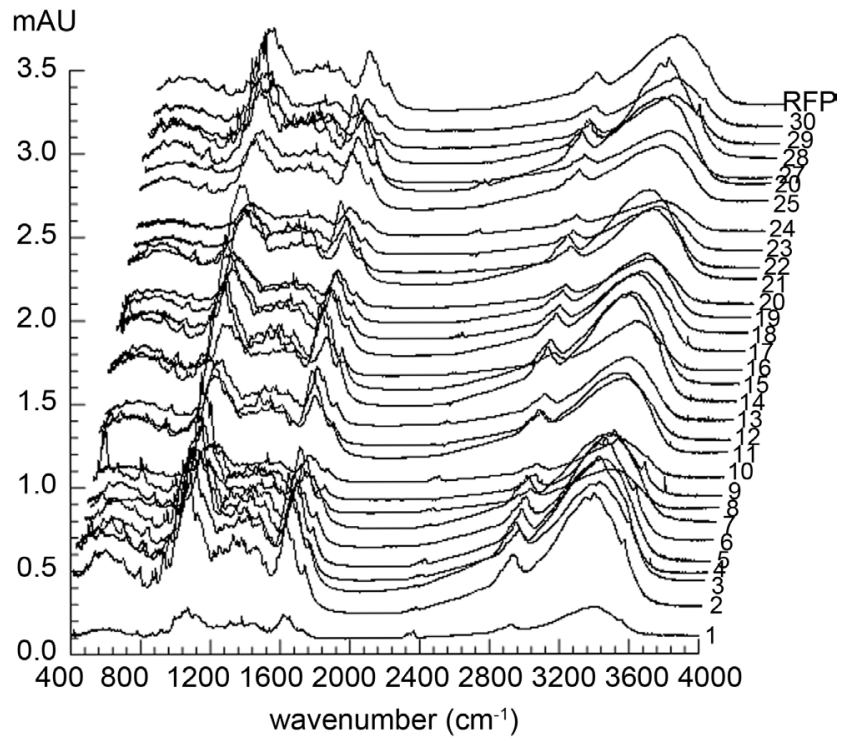

(b)

mAU

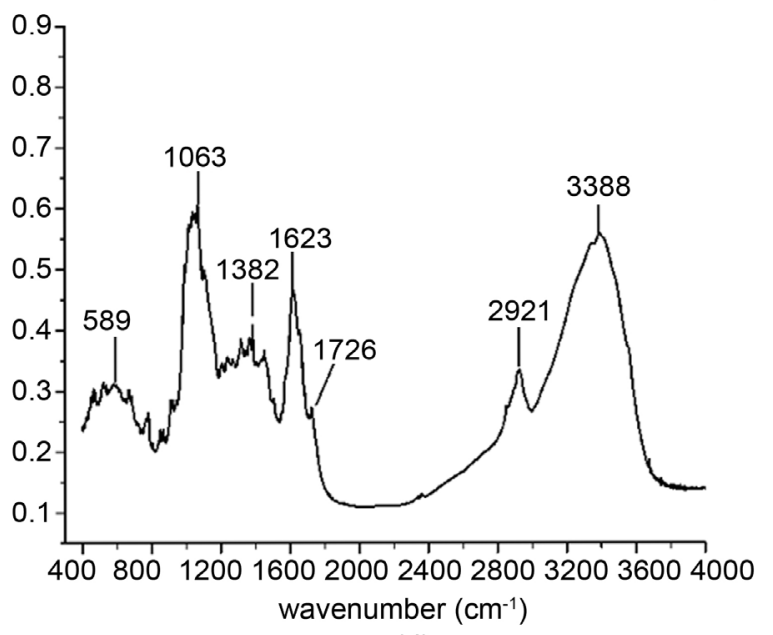

(d)

Figure 2. The spectral fingerprints of 30 batches of SHT and their reference fingerprints. (a): UV fingerprints; (b): IR fingerprints; (c): UV reference fingerprint; (d): IR reference fingerprint.

determined by analyzing the same sample solution six times consecutively. The sample solution stability was evaluated by analyzing a single sample stored in the dryer at room temperature for $0,0.25,0.5,0.75$ and 1.0 hour, and the results showed that $S_{\mathrm{IR}} \geq 0.99$ with RSD $\leq 1.0 \%$.

\subsubsection{Spectrum Analysis}

Nine-points smoothing technique was performed for original spectral data in order to remove unwanted variations and increase spectral resolution. The IR spectra fingerprints of thirty SHT samples from 4000 to $400 \mathrm{~cm}^{-1}$ and the RFP generated by averaging all of the spectra were shown in Figure 2(b). The variation of the collected samples was significant especially from 1500 to $400 \mathrm{~cm}^{-1}$ (mainly fingerprinting region) and several fingerprint characteristics were 
Table 3. The similarity analysis results in terms of UV, IR and CH by SQFM.

\begin{tabular}{|c|c|c|c|c|c|c|c|c|c|c|c|c|c|c|c|c|c|c|}
\hline & Para. & S1 & S2 & S3 & S4 & S5 & S6 & S7 & S8 & S9 & S10 & S11 & S12 & $\mathrm{S} 13$ & S14 & S15 & S16 & S17 \\
\hline \multirow{10}{*}{ UV } & $S_{\mathrm{UV}}$ & 0.99 & 0.99 & 0.98 & 0.98 & 0.97 & 0.99 & 0.97 & 0.95 & 0.97 & 0.99 & 0.96 & 0.99 & 0.98 & 0.99 & 0.97 & 0.98 & 0.98 \\
\hline & $P_{\mathrm{UV}}(\%)$ & 92.2 & 114.0 & 85.8 & 109.2 & 83.6 & 86.2 & 71.0 & 253.8 & 103.6 & 90.4 & 78.4 & 147.7 & 115.5 & 82.7 & 70.5 & 113.8 & 46.3 \\
\hline & $\alpha_{\mathrm{UV}}$ & 0.02 & 0.00 & 0.01 & 0.01 & 0.01 & 0.01 & 0.00 & 0.00 & 0.05 & 0.02 & 0.02 & 0.01 & 0.03 & 0.02 & 0.00 & 0.02 & 0.03 \\
\hline & Grade & 2 & 3 & 3 & 2 & 3 & 3 & 5 & 8 & 2 & 2 & 4 & 7 & 3 & 3 & 5 & 3 & 8 \\
\hline & Para. & S18 & S19 & S20 & S21 & S22 & S23 & S24 & S25 & S26 & S27 & S28 & S29 & S30 & RFP & \multicolumn{2}{|c|}{ Average } & RSD (\%) \\
\hline & $\mathcal{S}_{\mathrm{UV}}$ & 0.98 & 0.98 & 0.99 & 0.99 & 0.99 & 0.99 & 0.98 & 0.99 & 0.97 & 0.94 & 0.96 & 0.93 & 0.99 & 1.00 & \multicolumn{2}{|c|}{0.98} & 1.56 \\
\hline & $P_{\mathrm{UV}}(\%)$ & 227.3 & 78.2 & 145.5 & 66.3 & 67.5 & 96.6 & 127.3 & 66.5 & 76.8 & 54.1 & 176.2 & 102.4 & 171.3 & 100 & \multicolumn{2}{|c|}{106.69} & 45.33 \\
\hline & $\alpha_{\mathrm{Uv}}$ & 0.02 & 0.02 & 0.02 & 0.01 & 0.00 & 0.01 & 0.03 & 0.01 & 0.05 & 0.02 & 0.06 & 0.14 & 0.01 & 0.00 & \multicolumn{2}{|c|}{0.02} & 121.20 \\
\hline & Grade & 8 & 4 & 7 & 6 & 6 & 1 & 5 & 6 & 4 & 7 & 8 & 3 & 8 & 1 & \multicolumn{2}{|c|}{--} & -- \\
\hline & Para. & S1 & S2 & S3 & S4 & S5 & S6 & S7 & S8 & S9 & S10 & S11 & S12 & S13 & S14 & S15 & S16 & S17 \\
\hline \multirow{10}{*}{ IR } & $S_{\mathrm{IR}}$ & 0.96 & 1.00 & 0.99 & 1.00 & 0.99 & 1.00 & 1.00 & 0.99 & 0.94 & 0.99 & 0.99 & 1.00 & 0.99 & 1.00 & 1.00 & 0.99 & 0.99 \\
\hline & $P_{\mathrm{IR}}(\%)$ & 148.7 & 140.6 & 168.8 & 124.2 & 118.3 & 115.7 & 120.4 & 77.7 & 76.2 & 65.6 & 118.2 & 84.7 & 87.6 & 129.3 & 116.3 & 73.5 & 103.9 \\
\hline & $\alpha_{\mathrm{IR}}$ & 0.07 & 0.02 & 0.01 & 0.01 & 0.04 & 0.03 & 0.01 & 0.03 & 0.06 & 0.03 & 0.00 & 0.00 & 0.00 & 0.03 & 0.02 & 0.02 & 0.02 \\
\hline & Grade & 7 & 7 & 8 & 4 & 3 & 3 & 4 & 4 & 4 & 6 & 3 & 3 & 3 & 5 & 3 & 5 & 1 \\
\hline & Para. & S18 & S19 & S20 & S21 & S22 & S23 & S24 & S25 & S26 & S27 & S28 & S29 & $\mathrm{S} 30$ & RFP & \multicolumn{2}{|c|}{ Average } & RSD (\%) \\
\hline & $\mathcal{S}_{\mathrm{IR}}$ & 1.00 & 1.00 & 1.00 & 1.00 & 0.99 & 1.00 & 0.99 & 0.99 & 0.99 & 1.00 & 0.99 & 0.99 & 0.99 & 1.00 & \multicolumn{2}{|c|}{0.99} & 1.16 \\
\hline & $P_{\mathrm{IR}}(\%)$ & 87.4 & 83.0 & 67.8 & 113.2 & 72.2 & 68.7 & 67.1 & 111.0 & 140.1 & 76.4 & 102.4 & 74.7 & 74.7 & 100 & \multicolumn{2}{|c|}{100.28} & 28.21 \\
\hline & $\alpha_{\mathrm{IR}}$ & 0.00 & 0.01 & 0.01 & 0.02 & 0.02 & 0.01 & 0.05 & 0.04 & 0.01 & 0.02 & 0.04 & 0.01 & 0.01 & 0.00 & \multicolumn{2}{|c|}{0.02} & 79.15 \\
\hline & Grade & 3 & 3 & 6 & 3 & 5 & 6 & 6 & 3 & 7 & 4 & 1 & 5 & 5 & 1 & \multicolumn{2}{|c|}{--} & - - \\
\hline & Para. & S1 & S2 & S3 & S4 & S5 & S6 & S7 & S8 & S9 & S10 & S11 & $\mathrm{S} 12$ & $\mathrm{~S} 13$ & S14 & S15 & S16 & S17 \\
\hline \multirow{12}{*}{$\mathrm{CH}$} & $\begin{array}{c}Q_{V} \\
(\mathrm{~kJ} / \mathrm{g})\end{array}$ & 16.73 & 16.51 & 15.78 & 16.03 & 16.68 & 17.30 & 16.57 & 17.07 & 16.10 & 16.88 & 16.58 & 16.92 & 16.53 & 16.68 & 16.13 & 15.73 & 15.34 \\
\hline & $S_{\mathrm{q}}$ & 0.97 & 0.98 & 0.97 & 0.99 & 0.97 & 0.93 & 0.98 & 0.95 & 0.99 & 0.96 & 0.98 & 0.95 & 0.98 & 0.97 & 1.00 & 0.97 & 0.95 \\
\hline & $P_{\mathrm{q}}(\%)$ & 103.3 & 101.9 & 97.4 & 99.0 & 103.0 & 106.8 & 102.3 & 105.4 & 99.4 & 104.2 & 102.4 & 104.5 & 102.1 & 103.0 & 99.6 & 97.1 & 94.7 \\
\hline & $\alpha_{\mathrm{q}}$ & 0.03 & 0.02 & 0.03 & 0.01 & 0.03 & 0.07 & 0.02 & 0.05 & 0.01 & 0.04 & 0.02 & 0.05 & 0.02 & 0.03 & 0.00 & 0.03 & 0.05 \\
\hline & Grade & 1 & 1 & 1 & 1 & 1 & 2 & 1 & 2 & 1 & 1 & 1 & 1 & 1 & 1 & 1 & 1 & 2 \\
\hline & Para. & S18 & S19 & S20 & S21 & S22 & S23 & S24 & S25 & S26 & S27 & S28 & S29 & S30 & \multicolumn{2}{|c|}{ Average } & \multicolumn{2}{|c|}{ RSD (\%) } \\
\hline & $\begin{array}{c}Q_{V} \\
(\mathrm{~kJ} / \mathrm{g})\end{array}$ & 16.07 & 16.05 & 16.07 & 15.68 & 16.01 & 15.87 & 15.73 & 15.20 & 16.19 & 16.45 & 16.49 & 15.16 & 15.31 & \multicolumn{2}{|c|}{16.20} & \multicolumn{2}{|c|}{3.46} \\
\hline & $S_{\mathrm{q}}$ & 0.99 & 0.99 & 0.99 & 0.97 & 0.99 & 0.98 & 0.97 & 0.94 & 1.00 & 0.98 & 0.98 & 0.94 & 0.95 & \multicolumn{2}{|c|}{0.97} & & .97 \\
\hline & $P_{\mathrm{q}}(\%)$ & 99.2 & 99.1 & 99.2 & 96.8 & 98.9 & 98.0 & 97.1 & 93.8 & 100.0 & 101.6 & 101.8 & 93.6 & 94.6 & \multicolumn{2}{|c|}{100} & & .46 \\
\hline & $\alpha_{\mathrm{q}}$ & 0.01 & 0.01 & 0.01 & 0.03 & 0.01 & 0.02 & 0.03 & 0.06 & 0.00 & 0.02 & 0.02 & 0.06 & 0.05 & & .03 & & 7.80 \\
\hline & Grade & 1 & 1 & 1 & 1 & 1 & 1 & 1 & 2 & 1 & 1 & 1 & 2 & 2 & - & & & -- \\
\hline & Para. & S1 & S2 & S3 & S4 & S5 & S6 & S7 & S8 & S9 & S10 & S11 & $\mathrm{S} 12$ & S13 & S14 & S15 & S16 & S17 \\
\hline & $S_{\mathrm{I}}$ & 0.97 & 0.99 & 0.98 & 0.99 & 0.98 & 0.97 & 0.98 & 0.96 & 0.97 & 0.98 & 0.98 & 0.98 & 0.98 & 0.99 & 0.99 & 0.98 & 0.97 \\
\hline & $P_{\mathrm{I}}$ & 114.7 & 118.8 & 117.3 & 110.8 & 101.6 & 102.9 & 97.9 & 145.6 & 93.1 & 86.7 & 99.7 & 112.3 & 101.7 & 105.0 & 95.5 & 94.8 & 81.6 \\
\hline & $\alpha_{\mathrm{I}}$ & 0.04 & 0.01 & 0.02 & 0.01 & 0.03 & 0.04 & 0.01 & 0.03 & 0.04 & 0.03 & 0.01 & 0.02 & 0.02 & 0.03 & 0.01 & 0.02 & 0.03 \\
\hline & Grade & 3 & 3 & 3 & 3 & 1 & 1 & 1 & 7 & 2 & 3 & 1 & 3 & 1 & 1 & 1 & 2 & 3 \\
\hline & Para. & S18 & S19 & S20 & S21 & S22 & S23 & S24 & S25 & S26 & S27 & S28 & S29 & S30 & Avera & & RSI & D (\%) \\
\hline & $S_{\mathrm{I}}$ & 0.99 & 0.99 & 0.99 & 0.99 & 0.99 & 0.99 & 0.98 & 0.97 & 0.99 & 0.97 & 0.98 & 0.95 & 0.98 & & .98 & & .94 \\
\hline & $P_{\mathrm{I}}$ & 138.0 & 86.8 & 104.2 & 92.1 & 79.5 & 87.8 & 97.2 & 90.4 & 105.6 & 77.4 & 126.8 & 90.2 & 113.5 & & 2.32 & & 5.84 \\
\hline & $\alpha_{\mathrm{I}}$ & 0.01 & 0.01 & 0.01 & 0.02 & 0.01 & 0.01 & 0.04 & 0.04 & 0.02 & 0.02 & 0.04 & 0.07 & 0.02 & & .02 & & 6.78 \\
\hline & Grade & 6 & 3 & 1 & 2 & 4 & 3 & 1 & 2 & 2 & 4 & 5 & 2 & 3 & - & & & -- \\
\hline
\end{tabular}

RFP: Reference fingerprint; RSD: Relative standard deviation. 
revealed in Figure 2(d), the strongest peak at $3388 \mathrm{~cm}^{-1}$ indicated the stretching vibration of $\mathrm{O}-\mathrm{H}$ groups associated with phenolic hydroxyl of flavonoids and phenoliacids. The peak at $2921 \mathrm{~cm}^{-1}$ and $1382 \mathrm{~cm}^{-1}$ belonged to the stretching vibration of $-\mathrm{CH}_{2}$ and $-\mathrm{CH}_{3}$ groups, respectively, and the stronger peaks in the range of $1200-1000 \mathrm{~cm}^{-1}$ mainly attributed to the stretching vibration of $\mathrm{C}-\mathrm{O}$, which might be the characteristic absorptions of glycosides.

\subsubsection{Fingerprint Analysis in Terms of SQFM}

The IR spectra of all SHT samples and the RFP were imported into the in-house software to calculate similarity parameters $\left(S_{\mathrm{IR}}, P_{\mathrm{IR}}, \alpha_{\mathrm{IR}}\right)$ and final quality. It was demonstrated from Table 3 that although qualitative similarity $S_{\text {IR }}$ was able to slightly discriminate samples from batch to batch, a wide range of quantitative similarity $P_{\mathrm{IR}}$ led to final various quality grades. Most of the samples are qualified (grade $\leq 5$ ) except for S1-S3, S10, S20, S23, S24 and S26.

What's more, compared with the UV fingerprint, enormous difference has been found in some samples. A case in point was S8, instead of inferior (grade 8) in UV analysis, S8 was recognized as qualified (grade 4) with IR analysis. To our knowledge, it is mainly attributed to the different principles between IR and UV. UV fingerprint reveals the features of $\pi \rightarrow \pi^{*}, n \rightarrow \pi^{*}$ and $n \rightarrow \sigma^{*}$ chemical bonds of compounds while IR fingerprint chiefly reflects the vibration and rotation of chemical bonds (especially saturated bonds). It reminds us that multi-analysis methods based on diverse principles is necessary to comprehensively control the quality of TCM or HD.

\section{3. $\mathrm{CH}$ Analysis}

$\mathrm{CH}$ as a traditional parameter according to the First Law of Thermodynamics can disclose the total chemical energy in single compounds or mixture. $\mathrm{CH}$ can reflect not only the total chemical contents but also the therapeutic effect of TCM or HD to some extent [16] [17].

Consequently, it was introduced as an important approach in this study to analyze the quality of herbal medicines. The $\mathrm{CH}$ of thirty samples was determined using the calorimeter after instrument adjustment and precision validation. Qualitative similarity $S_{q}$, quantitative similarity $P_{q}$ and variation coefficient $\alpha_{q}$ were defined as Equations (4)-(6), of which $Q_{\mathrm{v}}$ was the $\mathrm{CH}$ value of each sample and $\bar{Q}_{\mathrm{v}}$ served as the reference value calculated by the average method.

$$
\begin{gathered}
S_{q}=1-\left|1-\frac{Q_{\mathrm{v}}}{\bar{Q}_{\mathrm{v}}}\right| \\
P_{q}=\frac{Q_{\mathrm{v}}}{\bar{Q}_{\mathrm{v}}} \times 100 \% \\
\alpha_{q}=1-S_{q}
\end{gathered}
$$

It was noticed that all samples had similar quality (grade 1 - 2) with qualitative 
similarity $S_{q} \geq 0.93$ and quantitative parameter $P_{q} \geq 93.6 \%$ shown in Table 3.

\subsection{Integrated Evaluation Involving UV and IR Fingerprinting as Well as $\mathrm{CH}$ Analysis}

In order to avoid bias detection at a single method, an integrated evaluation was carried out by means of equal weights. The integrated $S_{\mathrm{I}}, P_{\mathrm{I}}$ and $\alpha_{\mathrm{I}}$ values were calculated according to Equations (7)-(9).

$$
\begin{gathered}
S_{\mathrm{I}}=\frac{1}{3}\left(S_{\mathrm{UV}}+S_{\mathrm{IR}}+S_{q}\right) \\
P_{\mathrm{I}}=\frac{1}{3}\left(M_{\mathrm{UV}}+M_{\mathrm{IR}}+P_{q}\right) \\
\alpha_{\mathrm{I}}=\frac{1}{3}\left(\alpha_{\mathrm{UV}}+\alpha_{\mathrm{IR}}+\alpha_{q}\right)
\end{gathered}
$$

Our results illustrated that S8 and S18 were found to be outliers with the quality of grade 7 and 6 , respectively, the remaining twenty-eight samples had qualified grade in the range of 1 - 5 (specified in Figure 3 ).

\subsection{Comparative Analysis of Results Obtained from Multiple Methods}

Table 3 displays the discrepant quantitative similarity values of 30 batches of sample, a possible explanation of the result might be due to the variability in the raw material associated with a wide range of factors (e.g., climate, geographical location, harvest time, etc.) and variability in the manufacturing processes. As shown in Figure 4, the quantitative similarity of many samples exhibited large diversity, which reminded us that it was essential to adopt multi-approaches integration to disclose the quality information of TCM and HD.

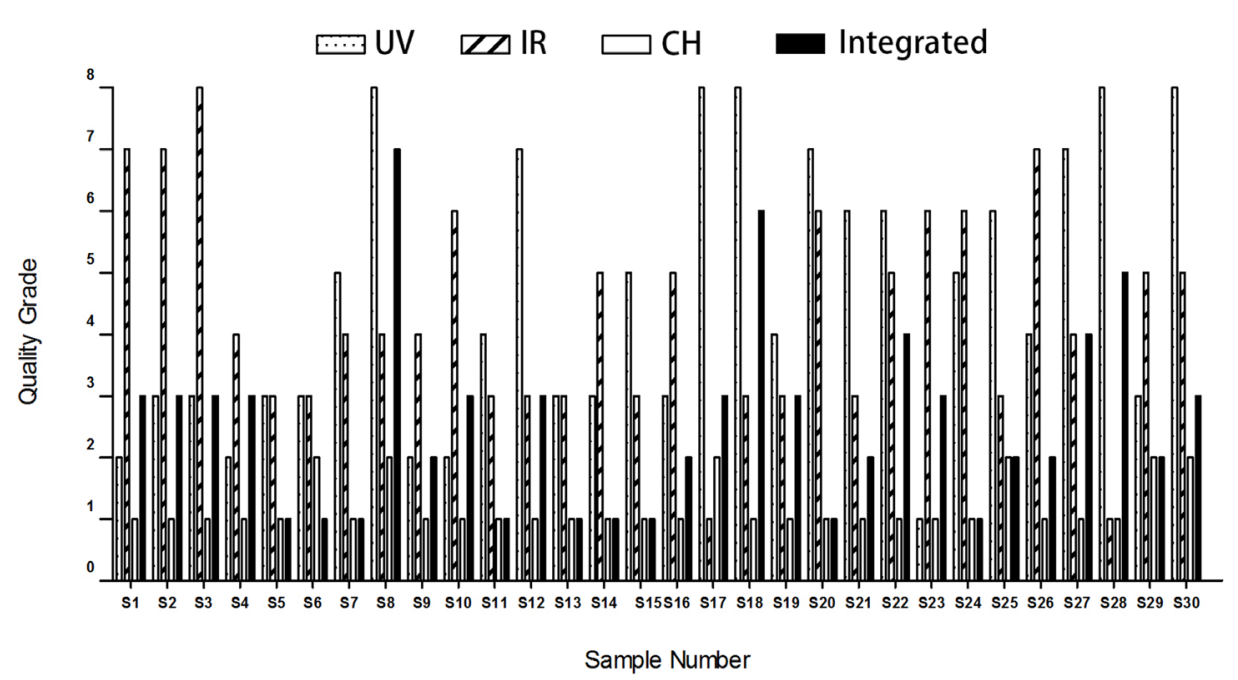

Figure 3. The quality grade of 30 samples identified by different methods. 


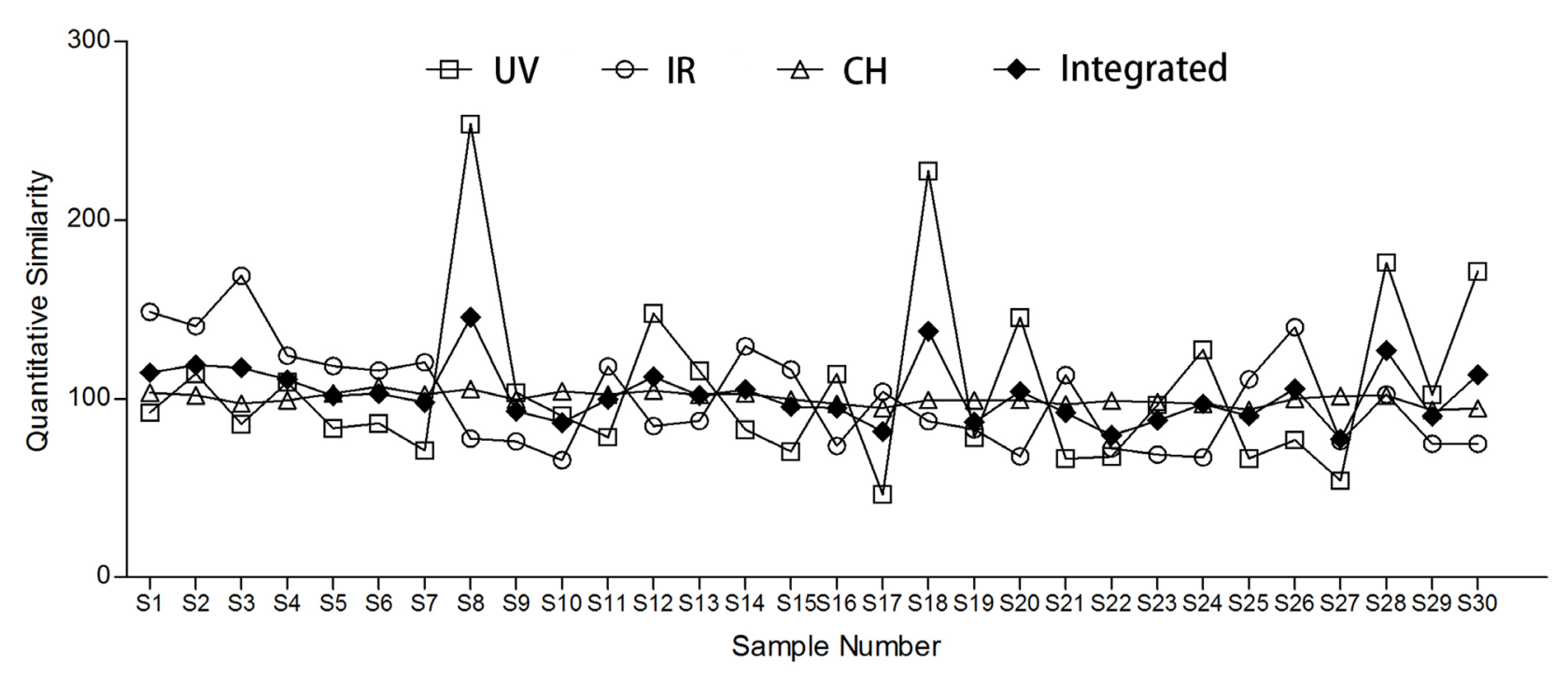

Figure 4. The comparison of quantitative similarity analysis for 30 batches of SHT in different analytical approaches calculated by SQFM.

\section{Conclusion}

Three approaches, UV and IR fingerprinting coupled with $\mathrm{CH}$ analysis, developed in the present study, represent fast and alternative way to traditional chromatographic analyses for the differentiation of SHT samples. They are all capable of acquiring data allowing discrimination of the quality consistency of herbal medicines from different sources. The promising results obtained from combination of multiple analytical methods encourage the application of the proposed strategy for the comprehensive quality assessment of raw herbal medicine and their preparations.

\section{Acknowledgements}

This research work was financially supported by the National Natural Science Foundation of China (Accession No. 81560695), and the Doctoral Research Fund of Dali University (Accession No. KYBS201512).

\section{References}

[1] Kong, W.J., Wang, J.B., Zang, Q.C., Xing, X.Y., Zhao, Y.L., Liu, W., Cheng, J., Li, Z.L. and Xiao, X.H. (2011) Fingerprint-Efficacy Study of Artificial Calculus bovis in Quality Control of Chinese Materia Medica. Food Chemistry, 127, 1342-1347. https://doi.org/10.1016/j.foodchem.2011.01.095

[2] Xie, P.S. and Leung, A.Y. (2009) Understanding the Traditional Aspect of Chinese Medicine in Order to Achieve Meaningful Quality Control of Chinese Materia Medica. Journal of Chromatography A, 1216, 1933-1940. https://doi.org/10.1016/j.chroma.2008.08.045

[3] Jiang, Y., David, B., Tu, P.F. and Barbin, Y. (2010) Recent Analytical Approaches in Quality Control of Traditional Chinese Medicines-A Review. Analytica Chimica Acta, 657, 9-18. https://doi.org/10.1016/j.aca.2009.10.024

[4] Valentina, R.N., Wannit, T.O., Benjamin, K., Qiao, V.L., Lin, B., Li, K.M., Roufoga- 
lis, B., Depo, Y., Meicun, Y. and Li, G.Q. (2010) Multiple Chromatographic and Chemometric Methods for Quality Standardisation of Chinese Herbal Medicines. Modernization of Traditional Chinese Medicine and Materia Medica, 12, 99-106.

[5] Ni, Y.N., Mei, M.H. and Kokot, S. (2012) One- and Two-Dimensional Gas Chromatography-Mass Spectrometry and High Performance Liquid Chromatography-Diode-Array Detector Fingerprints of Complex Substances: A Comparison of Classification Performance of Similar, Complex Rhizoma curcumae Samples with the Aid of Chemometrics. Analytica Chimica Acta, 712, 37-44.

https://doi.org/10.1016/j.aca.2011.11.010

[6] Napolitano, J.G., Godecke, T., Rodríguez-Brasco, M.F., Jaki, B.U., Chen, S.N., Lankin, D.C. and Pauli, G.F. (2012) The Tandem of Full Spin Analysis and qHNMR for the Quality Control of Botanicals Exemplified with Ginkgo biloba. Journal of Natural Products, 75, 238-248. https://doi.org/10.1021/np200949v

[7] Ni, L.J., Zhang, L.G., Hou, J., Shi, W.Z. and Guo, M.L. (2009) A Strategy for Evaluating Antipyretic Efficacy of Chinese Herbal Medicines Based on UV Spectra Fingerprints. Journal of Ethnopharmacology, 124, 79-86. https://doi.org/10.1016/j.jep.2009.04.006

[8] Yao, X., Peng, Y., Zhou, Q., Xiao, P.G. and Sun, S.Q. (2010) Distinction of Eight Lycium Species by Fourier-Transform Infrared Spectroscopy and Two-Dimensional Correlation IR Spectroscopy. Journal of Molecular Structure, 974, 161-164. https://doi.org/10.1016/j.molstruc.2010.02.064

[9] Li, D., Jin, Z.X., Zhou, Q., Chen, J.B., Lei, Y. and Sun, S.Q. (2010) Discrimination of Five Species of Fritillaria and Its Extracts by FT-IR and 2D-IR. Journal of Molecular Structure, 974, 68-72. https://doi.org/10.1016/j.molstruc.2010.01.031

[10] Lidia, E.A. (2011) Single Seed Discriminative Applications Using Near Infrared Technologies. Graduate Theses and Dissertations, 12023.

[11] Qin, K.M., Cai, H., Zheng, L.J., Zhang, M., Zhang, X.H., Gu, J. and Cai, B.C. (2013) Development of HPLC Fingerprint for Quality Assessment of Bulbus Lilii. Natural Product Communications, 8, 1447-1449.

[12] Zhao, Y.L., Fan, R.H., Yuan, H.X., Yu, M., Bi, K.S. and Yu, Z.G. (2011) Development of the Fingerprints for the Quality Evaluation of Viscum coloratum by High Performance Liquid Chromatography. Journal of Pharmaceutical and Biomedical Analysis, 1, 113-118. https://doi.org/10.1016/S2095-1779(11)70020-X

[13] Wang, Y., Sun, G.X., Liu, Z.B., Liu, Y.C., Gao, Y.N., Zhang, J.Q., Ji, Z.C. and Chen, X.X. (2014) Capillary Electrophoresis Fingerprinting Coupled with Chemometrics to Evaluate the Quality Consistency and Predict the Antioxidant Activity of Sanhuang Tablet as Part of Its Quality Control. Journal of Separation Science, 37, 3571-3578. https://doi.org/10.1002/jssc.201400765

[14] Wang, Y., Sun, G.X., Jin, Y., Xie, X.M., Liu, Y.C., Ma, D.D., Zhang, J., Gao, J.Y. and Li, Y.F. (2017) Holistic Evaluation of San-Huang Tablets Using a Combination of Multi-Wavelength Quantitative Fingerprinting and Radical-Scavenging Assays. Chinese Journal of Natural Medicines, 15, 310-320. https://doi.org/10.1016/S1875-5364(17)30049-3

[15] The State Pharmacopoeia Commission of China (2010) Pharmacopoeia of China. 2nd Edition.

[16] Cheng, J.T. (2000) Review: Drug Therapy in Chinese Traditional Medicine. Journal of Clinical Pharmacology, 40, 445-450. https://doi.org/10.1177/00912700022009198

[17] Cheng, D.H., Wang, J., Zeng, N., Xia, H.L., Fu, Y., Yan, D., Zhao, Y.L. and Xiao, 
X.H. (2011) Study on Drug Property Differences of Shexiang (Moschus) and Bingpian (Borneolum Synthcticum) Based on Analysis of Biothermodynamics. Journal of Traditional Chinese Medicine, 31, 21-26.

https://doi.org/10.1016/S0254-6272(11)60005-8 